\title{
Le Tramway de Claude Simon : les enseignements d'une expérience pédagogique
}

Metka Zupančič et Mojca Schlamberger Brezar

\section{(2) OpenEdition}

1 Journals

Édition électronique

URL : http://journals.openedition.org/ccs/949

DOI : $10.4000 /$ ccs.949

ISSN : 2558-782X

Éditeur :

Presses universitaires de Rennes, Association des lecteurs de Claude Simon

Édition imprimée

Date de publication : 30 avril 2015

Pagination : 79-92

ISBN : 9782753539990

ISSN : 1774-9425

Référence électronique

Metka Zupančič et Mojca Schlamberger Brezar, «Le Tramway de Claude Simon : les enseignements d'une expérience pédagogique », Cahiers Claude Simon [En ligne], 10 | 2015, mis en ligne le 22 septembre 2017, consulté le 21 avril 2019. URL : http://journals.openedition.org/ccs/949 ; DOI : $10.4000 /$ ccs. 949 


\title{
LE TRAMWAY DE CLAUDE SIMON : LES ENSEIGNEMENTS D'UNE EXPÉRIENCE PÉDAGOGIQUE
}

\author{
Metka ZUPANČIČ, \\ Université d'Alabama à Tuscaloosa, États-Unis \\ et Mojca SCHLAMBERGER BREZAR \\ Université de Ljubljana, Slovénie
}

\section{PROLOGUE}

Le projet que voici s'inscrit dans le cadre d'une recherche collaborative menée à l'Université de Ljubljana. En 2012, une subvention nationale slovène a permis au Département de traduction et d'interprétation de la faculté des Lettres d'accueillir Metka Zupančič, spécialiste de la littérature française contemporaine slovène qui œuvre à l'étranger, à l'université d'Alabama aux États-Unis. Dans la mesure où l'œuvre de Claude Simon occupe cette chercheuse depuis les années soixante-dix, compte tenu de son doctorat de troisième cycle soutenu à Strasbourg en 1977 et la continuation de ses recherches sur l'écrivain dans la thèse soutenue à Zagreb en $1988^{1}$, il paraissait intéressant de lui faire partager ses expériences dans un milieu où elle ne cesse d'être présente par ses travaux. En fait, c'est d'elle que viennent les premières traductions de $\mathrm{C}$. Simon dans cette aire géographique, non seulement en Slovénie mais dans le contexte de l'ancienne Yougoslavie où d'autres traductions, en serbe, en l'occurrence par Ivan Dimić, sont postérieures aux

1. Les deux thèses, retravaillées, ont été réunies en un volume, Lectures de Claude Simon: la polyphonie de la structure et du mythe, préf. M. Léonard, Toronto, GRÉF, 2001. 
siennes $^{2}$. Les deux romans traduits en slovène, Les Géorgiques en 1986 et La Route des Flandres en 1987, à la suite de l'attribution du Prix Nobel à l'écrivain, ont intéressé les éditeurs pour ces raisons précisément. Les Géorgiques s'imposait d'office pour une première traduction complète d'un roman simonien en slovène, vu que la deuxième thèse de 1988 portait principalement sur ce texte ${ }^{3}$. La publication a été intégrée dans la série des œuvres de Prix Nobel auprès de la maison d'édition Cankarjeva založba de Ljubljana, un des grands projets visant à familiariser le public slovène avec les titres imposants de la littérature mondiale; aujourd'hui, ni la série ni la maison d'édition n'existent malheureusement plus. Mais la qualité incontestable de l'œuvre simonienne a suscité l'intérêt d'un autre éditeur, Založba Obzorja, à Maribor, qui a accueilli La Route des Flandres . $^{4}$

Depuis, comme cela paraît être le cas en général pour une littérature considérée comme difficile face à la production littéraire plus récente, l'œuvre simonienne n'a pas fait l'objet de nouvelles traductions ni de nouvelles recherches en Slovénie. Toujours est-il qu'au début des années 80, période où Metka Zupančič a en quelque sorte introduit l'œuvre de Claude Simon en Slovénie, d'abord par quelques articles tirés de sa thèse de $3^{\mathrm{e}}$ cycle et la traduction des extraits de certains romans ${ }^{5}$, ce genre de littérature intéressait surtout un milieu relativement restreint d'intellectuels slovènes, écrivains et philosophes francophiles qui tenaient à ce que les courants littéraires français de pointe soient présents dans le contexte slovène. Il paraît intéressant qu'en 1986, deux élèves du lycée de Celje, Jasna Krajinović et Daša Orešnik ${ }^{6}$, aient choisi de travailler sur La Route des Flandres dans un projet de jeunes chercheurs (mais l'exploration n'a pas été prolongée). On comprend facilement qu'un quart de siècle plus tard, les nouvelles générations de lecteurs et traducteurs potentiels considèrent l'écriture

2. M. Zupančičc, "Réception: l'Europe de l'Est », Dictionnaire Claude Simon (dir. M. Bertrand), Honoré Champion, 2013, p. 882-883.

3. C. Simon, Georgike, trad. M. Zupančič, Ljubljana, Cankarjeva založba, 1986. [Les Géorgiques, Minuit, 1981.]

4. C. Simon, Flandrijska cesta, trad. M. Zupančič, Maribor, Obzorja, 1987. [La Route des Flandres, Minuit, 1962.]

5. M. Zupančič, l'ensemble thématique "Claude Simon ", introduction et traduction des extraits: Leçon de choses, "Tradition et révolution" et Orion aveugle; l'essai "Claude Simon in orfizem ", Nova revija, vol. V, $\mathrm{n}^{\circ}$ 48-49, 1986, p. 498-526. Voir aussi les articles suivants de M. Zupančič: "Prepletanje pomenov in oblikovnih značilnosti v Simonovi Flandrijski cesti », Vestnik, vol. 21, n 1-2, 1987, p. 46-56; "Triptih kot strukturno vodilo pri Šeligu in Simonu ", Primerjalna književnost, vol. VI, n ${ }^{\circ} 1,1983$, p. 34-43; " La réception du Nouveau Roman français en Slovénie ", Acta Neophilologica, vol. XXI, 1988, p. 39-51; "Les générateurs picturaux dans l'écriture simonienne ", Acta Neophilologica, vol. XV, 1982, p. 105-112.

6. J. Krajinović et D. Orešnik, Linterprétation des séries thématiques et des symboles sur la base d'associations dans La Route des Flandres de Claude Simon, sous la tutelle de M. Zupančič et S. Deržek. Celje, Srednja družboslovna šola, 1986. 
simonienne comme l'une des plus difficiles de la production littéraire française contemporaine. D'ailleurs, telle est aussi la perception de cette œuvre parmi les étudiants des cycles avancés à l'Université d'Alabama où enseigne M. Zupančič.

L'objectif principal de l'investigation présentée ici était donc d'examiner la pérennité des paradigmes véhiculés par l'écriture simonienne, ainsi que la possibilité de leur saisie par les nouvelles générations de lecteurs et de futurs traducteurs. L'expérience menée avec les étudiants a ainsi permis de relever le défi d'une traduction partielle d'un passage de haute complexité, et ce, en comparaison avec la démarche effectuée à la même période dans une classe de littérature française à l'Université d'Alabama. Voici donc les résultats qui peuvent être tirés de cette expérience pédagogique partagée.

\section{LES CONDITIONS ET LES OBJECTIFS DE L'EXPÉRIENCE}

À la suite d'une conférence de M. Zupančič, à l'Université de Ljubljana, au printemps 2011, sur les enjeux majeurs de sa propre traduction des Géorgiques, l'idée initiale était de faire traduire aux étudiants un passage de ce même roman et ensuite d'effectuer une comparaison avec la première traduction de 1986, pour établir certains paramètres traductologiques et vérifier en quoi l'évolution de la langue slovène, dans ces quelques vingt-cinq années, pouvait avoir affecté la nouvelle approche des traducteurs. Par la suite, dans un cours sur les Prix Nobel de littérature française, cette fois à l'université d'Alabama, au printemps 2012, M. Zupančič a proposé à sa classe américaine plutôt l'étude du Tramway, dans la mesure où il s'agit du dernier roman publié du vivant de l'auteur et qui, par ailleurs, représente la somme de tous les thèmes majeurs de cette écriture. Une version condensée de ce cours, sous forme d'une conférence à l'université de Ljubljana en juin 2012, avec l'accent mis justement sur Le Tramway simonien, a ensuite permis un travail de traduction dans cet axe.

Pour mieux assurer la comparaison entre les niveaux de réception du texte, de la part des étudiants des deux universités, un même passage du Tramway, l'extrait qui se situe entre les pages 62 et $64^{7}$ de l'édition Minuit, a été analysé. Cet extrait est particulièrement exigeant puisqu'il offre la condensation de plusieurs ensembles thématiques, marques de l'intertextualité interne de l'œuvre, et favorise la rencontre de plusieurs « séries » narratives. En outre, l'extrait est placé presque au milieu du livre et représente ainsi un passage charnière, une mise en

7. C. Simon, Le Tramway, Minuit, 2001, p. 62-64. De "Vieillard " (p. 62) à " une sorte de sénilité du rouge " (p. 64). 
abyme du roman entier, comme cela est suggéré par le trajet du tramway (comme motif et comme métaphore) entre plusieurs mouvements textuels.

Alors que plusieurs groupes d'étudiants de l'université de Ljubljana s'étaient déjà penchés sur la traduction de cet extrait et qu'une étudiante américaine avait bien voulu relever le même défi, une conférence supplémentaire sur l'écriture simonienne à la faculté des Lettres de Ljubljana, en janvier 2013, a permis de mettre en lumière les points principaux qui devaient être reconsidérés dans la traduction de l'extrait choisi.

La traduction du passage en classe de traduction du français vers le slovène a été proposée aux niveaux Master $1(\mathrm{bac}+4)$ et Master $2(\mathrm{bac}+5) \mathrm{du}$ Département de traduction et d'interprétation de l'université de Ljubljana, dont seule une minorité, de niveau Master 2, a suivi la conférence de M. Zupančič sur les Prix Nobel français, tandis que les étudiants du Master 1 se sont lancés dans la traduction sans préparation théorique au texte choisi. Toutefois, précisons que tous les étudiants slovènes avaient suivi le cours de théorie de la traduction ainsi que le cursus sur la traduction littéraire. On pouvait alors s'attendre à des connaissances de base dans le domaine de la traductologie et aussi à un choix adéquat des stratégies dans l'approche de la traduction.

Nous avons obtenu cinq traductions de niveau Master 2 et seize du niveau Master 1, au total vingt-et-une traductions de l'extrait vers le slovène. La traduction du français vers l'anglais a été préparée par une étudiante américaine qui était d'abord inscrite en quatrième année d'études et qui est passée au niveau de maîtrise, à l'université d'Alabama ${ }^{8}$. Nous avons donc pu comparer certains résultats de l'exercice pour savoir si les mêmes éléments de l'œuvre se sont montrés problématiques.

\section{LES POSTULATS DE DÉPART}

En nous référant à notre pratique ainsi qu'à nos connaissances de la structure des langues slovène et française, nous avons établi plusieurs postulats qui anticipaient en quelque sorte les difficultés qui pourraient survenir. Parmi ces points ardus, un aspect particulier de l'écriture simonienne et plus généralement

8. Il faudrait préciser que les étudiants américains à l'université d'Alabama ne suivent pas de cursus particulier en traductologie et qu'ainsi, tout travail d'initiation à la traduction se fait indirectement, par le biais de l'analyse textuelle dans le cadre de cours thématiques. De plus, pour les étudiants du $3^{\text {e }}$ cycle, l'anglais n'est pas nécessairement la langue maternelle. Pour toutes ces raisons, une expérience menée conjointement dans les deux universités et impliquant le groupe américain au complet ne s'avérait pas possible. Toutefois, l'étudiante américaine qui a bien voulu relever le défi de l'exercice était au courant du projet et a accepté que sa traduction fasse l'objet d'une analyse comparative. 
de celle du Nouveau Roman n'a peut-être pas été immédiatement compris par les lecteurs, même s'il affecte l'appréhension des dimensions de base de cette écriture. En principe, le narrateur porteur de la diégèse reste comme extérieur à tout processus mental imaginé ou pressenti auprès des actants dont il relate les expériences. Autrement dit, à la différence du narrateur traditionnel (qui revient dans la production littéraire contemporaine), celui qui s'exprime chez Simon n'éclaircit jamais les pensées de ceux ou celles qui font irruption dans le récit.

D'autres postulats sont liés à la structure linguistique, à savoir au passage difficile entre les deux langues. Il s'agit en premier lieu de l'allègement syntaxique dû au gérondif et, en second lieu, du passage du nominal au verbal, entre le français et le slovène, ainsi que du choix lexical approprié dans la langue cible, surtout pour des termes qui peuvent apparaître comme typiquement simoniens.

Dans l'extrait du Tramway que nous avons choisi pour la traduction, le style simonien ressort dans toute sa complexité: la première phrase, très longue, remplissant presque toute la page et mise en valeur par l'anacoluthe, est une phrase simonienne par excellence. Ce type d'expression exige nécessairement un effort intellectuel de la part du lecteur comme du traducteur à cause des multiples actants que ce fragment met en scène et qui s'entremêlent.

Ces postulats relèvent de la norme de traduction qui entre en vigueur dans la traduction littéraire (telle qu'elle a été présentée pour la première fois par Gideon Toury) ainsi que des normes fonctionnelles de la langue slovène. Selon G. Toury ${ }^{9}$, la norme traductive implique les valeurs générales et partagées par un groupe ${ }^{10}$. Les normes résultent d'ailleurs de l'intériorisation des contraintes ${ }^{11}$ et, de ce fait, dépendent aussi du traducteur. Et comme la norme traductionnelle va varier selon le type de texte ou bien selon la date de la traduction, il est impossible de définir une norme universelle qui nous suggèrerait une traduction idéale, en quelque sorte modèle.

La norme linguistique fonctionnelle en slovène, celle qui gère par exemple l'usage du gérondif et du participe présent, impose quant à elle, des contraintes structurelles. L'utilisation de ces structures, si fréquentes en français, est impossible dans quelques cas, notamment quand il s'agit d'une anacoluthe.

9. G. Toury, In Search of a Theory of Translation, Tel Aviv, Tel Aviv University - The Porter Institute for Poetics and Semiotics, 1980; Descriptive Translation Studies and Beyond, Amsterdam/Philadelphia, John Benjamins, 1995, p. 199.

10. Voir F. Plassard, "Aspects de la norme en traduction », Scolia, vol. 25, 2011 ("Traduction des normes et normes de traduction dans l'espace européen. Institutions et entreprises ", dir. T. Grass).

11. Ibid., p. 17. 
Dans les traductions vers le slovène, il faut aussi prendre en considération et assumer les contraintes stylistiques liées à l'usage des structures gérondives et participiales: il faut savoir que celles-ci étaient utilisées régulièrement dans la littérature slovène du XIX ${ }^{e}$ siècle tandis qu'aujourd'hui elles sont plutôt vieillies et peu usitées ${ }^{12}$, encore que structurellement admises.

\section{LES PRINCIPAUX DÉFIS DE LA TRADUCTION}

Alors que les contraintes structurelles liées au fonctionnement de la langue slovène sont à respecter impérativement, les contraintes stylistiques, liées à l'expression, quant à elles, peuvent être contournées ou changées. Quand il s'agit de traduction littéraire, d'autres postulats et normes peuvent entrer en jeu et entraîner une disparité entre la norme linguistique et la norme traductionnelle.

Cette disparité a été confirmée par la théorie de traductologie allemande des années 70 appelée "skopos ", élaborée par les chercheurs Hans Vermeer et Katharina Reiss, et plus particulièrement par cette dernière qui a mis en valeur la fonction du texte devant guider la traduction. Sa théorie est élaborée à la base des fonctions du langage de Karl Bühler, à savoir 1) la représentation des objets et des phénomènes; 2) l'attitude du producteur du texte à l'égard de ces objets et phénomènes; 3) l'adresse de l'auteur au récepteur du texte. K. Reiss prévoit ainsi au moins trois approches de la traduction, basées sur les trois types de textes, dénommés selon la fonction prédominante, à savoir les textes informatifs, expressifs et appellatifs (ou opérationnels, selon la traduction vers le français ${ }^{13}$ ). Dans les textes littéraires, c'est la fonction expressive qui est privilégiée. Celle-ci se rattache grosso modo à la fonction poétique, telle qu'elle est proposée par Roman Jakobson ${ }^{14}$ et qui met en valeur la structure stylistique de la phrase comme porteuse de signification. Lors de la traduction de ce type de textes, il faut donc respecter le point de vue expressif avant la fonction informative: le style est premier. Selon cette optique, même la solution qui garderait dans la langue cible une structure gérondive et participiale serait alors adéquate.

12. M. Jesenšek, Deležniki in deležja na -č in -ši, Maribor, Slavistično društvo, 1998.

13. K. Reiss, La critique des traductions, ses possibilités et ses limites: catégories et critères pour une évaluation pertinente des traductions, trad. C. Bocquet, Artois, Artois Presses Université, "Cahiers de l'Université d'Artois ", 2002 (1976); voir aussi M. Guidère, Introduction à la traductologie: penser la traduction, hier, aujourd'hui, demain, Bruxelles, De Boeck, 2008.

14. R. Jakobson, Essais de linguistique générale, trad. et préf. N. Ruwet, Minuit, 1963. 


\section{IDENTIFIER LES ACTANTS}

Pour l'extrait du Tramway choisi, à titre d'exemple des difficultés que représente l'écriture simonienne pour tous ceux qui s'efforcent de la transposer dans une autre langue, une analyse détaillée s'impose. Après un premier regard sur la structure syntaxique, il paraissait impératif d'établir d'abord l'identité des " actants " évoqués dans ce fragment textuel, pour ensuite pouvoir mieux saisir les méandres par lesquels l'écrivain parvient à établir une accumulation d'analogies basées sur plusieurs métaphores filées qui, si on les étudie de près, se combinent aussi avec une série de métonymies ${ }^{15}$.

\section{Vieillard - parallèle maman - parallèle prisonnier}

Parmi les éléments du lexique, si on ne distingue pas bien les nuances ou même les degrés d'intensité des qualifiants, comme dans le cas des adjectifs servant à désigner des couleurs, de nombreux problèmes peuvent surgir lors de la traduction. En l'occurrence, les couleurs s'inscrivent dans l'isotopie qui domine ce passage - la rencontre avec la mort, sous des aspects variés -, en créant des liens métaphoriques et des allusions qui pointent vers d'autres éléments du discours.

À observer ce qui se passe au niveau des actants, il s'agit d'un parallèle mais également d'un contraste entre le "vieillard ", introduit sans aucun déterminant au début du paragraphe (p. 62) et "maman », donc, entre deux représentations de la maladie et de l'approche de la mort. Si l'allure du vieillard est définie par une forme d' " indécence » et une sorte d'obscénité, la mère atteinte de cancer est associée, elle, à une certaine " décence ", par exemple à travers le port de "cette perruque aux ondulations figées" (p. 63) - où l'adjectif " figées " signale déjà un certain éloignement par rapport à la vie humaine (une immobilisation du mouvement suggérée aussi par l'évocation de "cet aspect guignolesque et macabre de marionnette ", p. 63). Le « moi-je » est ici pleinement présent, mais ne dit ni son nom ni son âge à l'époque de l'hospitalisation, alors que cette situation permet, elle, une concaténation mémorielle de retours en arrière: c'est lui qui émet des jugements de valeur (sur l'obscénité, sur l'indécence du voisin de chambre) et qui voit derrière la personne en face de lui ou derrière le souvenir de la mère une série d'autres images, en commençant par celles d'oiseaux rapaces.

15. La complexité de cet enchâssement de métaphores à l'intérieur des métonymies est apparue fascinante, même si elle a été extrêmement difficile à décoder lorsque le texte a été analysé à l'université d'Alabama. Les étudiants ont reconnu avoir acquis des outils non seulement pour la lecture de C. Simon mais pour la littérature française en général. 
S’y ajoute un élément qui n'a rien à voir ni avec le vieillard à l'hôpital ni avec la mère, mais amené par l'analogie avec la métaphore de la marionnette: l'image du prisonnier de guerre humilié et injurié, puni d'avoir volé le pain de ses camarades. L'accumulation des métaphores s'intègre à ce qu'on pourrait identifier comme une métonymie géante où tous les éléments font partie d'un grand tout. Une nouvelle opposition se manifeste alors, entre la maladie ou la vieillesse - à savoir entre les forces « invisibles» qui détruisent l'être humain, et une force humaine - mais qui devient inhumaine - celle des Allemands qui imposent la pire des punitions pour un acte de désespoir face au dénuement total.

\section{LES EFFETS DU TEXTE}

Venons-en à l'analyse des termes qui, de prime abord, ont suscité une difficulté pour la traduction. Il est intéressant de noter que les points problématiques se sont dessinés justement autour des mots clés de l'écriture simonienne.

\section{L'espace de «transit»}

Le premier mot était " transit " désignant le lieu où les malades, dans l'hôpital, attendaient leur transfert vers un autre département spécialisé. On doit souligner ici le sens particulier attribué à ce lieu; en effet, le dictionnaire TLF ne donne pas de sens pour le mot "transit " dans un contexte de ce type; l'emploi le plus proche est le suivant: "Cité de transit. Lieu d'hébergement provisoire où sont logés des immigrés ou des réfugiés" (Dict. $\mathrm{Xx}^{\mathrm{e}} \mathrm{s}$. $)^{16}$. Quelques étudiants ont traduit ce mot par " čakalnica ", c'est-à-dire "salle d'attente ", ou alors ont laissé l'équivalent du terme français, le mot " tranzit ». Mais le sens du tranzit en slovène est lié au transport (ininterrompu) à travers un État ou une région (selon SSKJ ${ }^{17}$. C'est un mot doublement problématique, puisqu'il revêt déjà un sens atypique en français. En plus, dans ce contexte, il renvoie manifestement à l'étymologie latine de transire; l'on songe à l'expression chrétienne sic transit gloria mundi, "Ainsi passe la gloire du monde ", soulignant le passage vers la mort et la détérioration. Le mot est aussi mis en valeur par son aspect orthographique, étant écrit en majuscules.

16. En ligne, atilf.atilf.fr.

17. A. Bajec et al., Slovar slovenskega knjižnega jezika (SSKJ), Ljubljana, DZS, 1994 - le grand dictionnaire monolingue de la langue slovène. 


\section{« Le bec de rapace » et « la perruque aux ondulations figées "}

Un autre signe de la détérioration (voire de la dégradation) des figures évoquées est le bec de rapace comme métaphore pour le nez du vieillard aminci par la maladie, puis de la mère du narrateur, comme par exemple dans le syntagme suivant: «encadrant un semblable et tragique visage au bec de rapace ". Ce bec de rapace contraste avec la perruque aux ondulations figées, qui vise à dissimuler les marques de la maladie puisqu'elle réintroduit le mouvement dans le visage maternel. Mais précisément, la notation « ondulations figées » était opaque pour les étudiants: ils parlaient de «la perruque aux fausses ondulations " (lasulja $z$ nepravimi kodri) ou aux ondulations drues (trdi kodri) ou de la perruque à ondulation permanente (lasulja s trajno).

Simon suggère que cet effort pour masquer les signes de la maladie ne fonctionne pas, car la chevelure permanentée n'est pas mouvement. Elle annonce le figement du visage. Ces jeux de nuance qui passent par un simple adjectif connoté sont complexes.

\section{Polichinelle, Guignol, marionnette}

Le Polichinelle comme personnage de la commedia dell'arte n'est pas aussi connu dans la culture slovène que dans la culture française. Ce personnage, mentionné dans l'extrait, a été remplacé dans une grande partie des traductions par l'équivalent culturel slovène Pavliha, qui représente un personnage avec beaucoup d'humour, qui brouille les situations mais finit par se tirer d'affaire indemne. C'est une équivalence acceptable. La traduction du " guignolesque" est plus difficile. Les étudiants qui ont fait de bons choix de traduction ont lié l'adjectif " guignolesque " à la comédie, voire au grotesque, en proposant la traduction komedijantski, grotesken. Ce lien symbolique entre le Polichinelle et la «marionnette " ne pose pas de difficultés car ce terme culturel spécifique est entré depuis longtemps dans le vocabulaire slovène.

\section{Adjectifs qui signalent la détérioration d'un corps et adjectifs de couleurs}

Des termes comme "visage fripé et déchu de Polichinelle » ne présentaient pas de problème particulier. En revanche, les adjectifs dénotant ou connotant des couleurs ont suscité maintes représentations subjectives chez les étudiants. La couleur argentine du syntagme " chevelure argentine " (p. 63) a été traduite cheveux argentins (en provenance d'Argentine), en slovène argentinski lasje, avec 
l'explication ou la supposition que C. Simon aurait fait référence à une chevelure noire et abondante. Il est intéressant de constater que cette erreur a été commise aussi bien par quelques étudiants slovènes que par l'étudiante américaine qui s'est prêtée à l'exercice de la traduction vers l'anglais.

Les autres adjectifs qui, dans l'extrait choisi, évoquent les couleurs vont du bleu marine au rouge théâtral, avec la « symphonie d'orangés, de verts et d'une couleur qui n'était pas le rose mais comme un vieillissement, une sorte de sénilité du rouge " (p. 64). Les nuances de couleurs ont été bien maîtrisées. Seul le rouge théâtral a été mal rendu en slovène (rouge " criard »). Une traduction a même tenté l'équivalent slovène du rouge dramatique par association au "théâtre ». Il s'agit ici d'une transposition de sens : du lieu on passe à l'action associée à ce lieu, ce qui signale un effet de "surtraduction".

\section{Les modalisateurs}

On peut considérer que la narration du Tramway toute entière est plongée dans le vague, dans l'incertitude. Simon emploie les modalisateurs du nom qui déstabilisent le sens et l'assurance de la lecture, par exemple en usant des tournures une sorte de, un certain, c'est-à-dire. À titre d'exemple, voici les syntagmes: " une sorte de double ricanant de moi-même "; " c'est-à-dire ce misérable acharnement "; " une résistance pour ainsi dire obscène ».

Les connecteurs de comparaison et de reformulation (ainsi que, comme, non pas... que, tel, comme si, c'est-à-dire, parmi d'autres) suggèrent le vague et l'indécis. Ils ont été facilement identifiés comme effets stylistiques particuliers de l'écriture simonienne et ont été respectés dans la traduction. S'ajoutent à ces modalisateurs des syntagmes comme "non que... mais peut-être", "paradoxalement, parce que ", qui n’ont pas semblé poser de problèmes aux étudiants. Voici l'exemple d'un court extrait significatif à cet égard, suivi d'une de ses traductions vers le slovène. Dans les deux cas, les modalisateurs sont en italiques:

mais peut-être, paradoxalement, parce que sa personne semblait incarner jusqu’à la caricature une résistance pour ainsi dire obscène à ce pour quoi nous nous trouvions tous les deux-là, comme s'il m'était donné de voir et de cohabiter avec une sorte de double ricanant de moimême, c'est-à-dire ce misérable acharnement qu'il mettait non seulement à vivre mais à nier une déchéance qu'il incarnait jusqu'à un insupportable degré d'indécence, passant et repassant sans cesse ce petit peigne dans la longue chevelure argentine qui semblait elle-même jurant avec le visage fripé et déchu de polichinelle sur le crâne duquel elle brillait comme un accessoire postiche, comme cette perruque aux ondulations figées que non par coquetterie bien sûr mais par un souci de décence (et sans doute pour m'épargner une vision trop effrayante) maman (je le sus plus tard) s'obstinait à porter, [...] 
ampak paradoksalno mogoče zato, ker je bilo, kot bi ta oseba do potankosti utelešala odpor, tako rekoč obscen, proti temu, zaradi česar sva bila oba tam, kot bi mi bilo dano videti in prebivati $\mathrm{z}$ nekakšnim svojim dvojnikom, ki se mi posmehuje, se pravi ta nesrečna zagrizenost, s katero je ne le živel, ampak tudi zanikoval nek propad, ki ga je utelešal do nemogoče mere nespodobnosti, tako da si je s tem majhnim glavnikom neprestano česal dolge srebrnosive lase, ki so bili $\mathrm{v}$ neskladju z zgubanim in propadlim pavlihastim obrazom in se na njegovi lobanji svetili kot nek pozneje dodani okrasek in bili videti kot tista lasulja z nepremičnimi kodri, ki jo je mama (to sem izvedel pozneje), seveda ne ker bi bila koketna, ampak iz skrbi, da bi bila spodobna (in nedvomno da bi mi prihranila kakšen preveč strašljiv prizor) trmasto nosila [...]

\section{L'ANACOLUTHE, EXEMPLE DE DIFFICULTÉ DE CONSTRUCTION MAJEURE}

L'anacoluthe en grammaire française (par exemple selon Maurice Grevisse $)^{18}$ est présentée tantôt comme un trope, tantôt comme un écart par rapport à la norme. En tant que trope, il sert à l'allègement syntaxique et présente une accélération discursive par le changement du sujet.

C'est par rapport aux actants (en tant que moteurs textuels) que surviennent les difficultés majeures au niveau de l'analyse syntaxique, car ils deviennent les actants du cadre verbal et influencent le changement de la structure syntaxique, comme dans l'exemple de l'anacoluthe "éprouvant" (au début du texte), avec un participe qui ne se rattache pas au sujet ("Vieillard»). Les participes présents " passant, repassant " et "incarnant " relèvent du même problème d'interprétation. Ainsi, l'anacoluthe "éprouvant immédiatement à son égard " souligne le passage de la pensée de l'auteur d'un actant à l'autre à l'intérieur d'une même phrase, ce qui entraîne le changement du sujet grammatical et parallèlement une réorientation du flux de pensée. Il semble que l'auteur emploie l'anacoluthe pour suggérer l'apparition brutale d'un nouvel actant.

Ce type de construction phrastique n'est pas facilement transposable d'une langue à l'autre et elle est impossible en slovène. Par conséquent, dans ce cas précis, il a fallu faire comprendre aux étudiants que cette construction devait être explicitée par une phrase juxtaposée: «takoj sem do njega začutil nepremostljiv odpor " (traduction littérale vers le français: "j’ai immédiatement éprouvé à son égard..."). 


\section{LE PARTICIPE PRÉSENT}

Certains participes présents et gérondifs forment aussi le noyau verbal de la subordonnée. Ils représentent un allègement syntaxique en français tandis qu'en slovène le traducteur procède par l'explicitation. L'explicitation des participes présents est le plus souvent rendue par la modulation temporelle, causale, ou bien par le recours à la coordination copulative ou à une subordonnée relative.

Ainsi, le syntagme «passant, repassant le petit peigne dans sa chevelure argentine " s'exprime en slovène par l'explicitation temporelle, voire par un verbe conjugué après une conjonction de temps et une tournure signalant la répétition: "ko si je z glavnikom kar naprej česal... ». Le participe présent « incarnant " subit quant à lui l'explicitation relative: "ki je utelešal».

Stylistiquement, une phrase participiale en slovène est plus marquée qu'une relative et l'accumulation de plusieurs relatives au cours d'une seule phrase ne pose pas de problèmes d'ordre stylistique ou syntaxique. Tout de même, il faut signaler que quelques étudiants-traducteurs ont senti que le remplacement des participes par des phrases relatives était une trahison du sens et ont opté pour la traduction avec participe, lorsque celle-ci était grammaticalement acceptable. En effet, le participe peut rester noyau phrastique si les sujets sont identiques. Et finalement, dans la traduction, le maintien du participe apporte son tribut à l'effet de style simonien.

\section{LA SYNTAXE SIMONIENNE}

Suite à des digressions de la pensée de l'auteur, la phrase simonienne présente un grand défi pour le lecteur ainsi que pour le traducteur. La phrase principale est divisée en subordonnées et en syntagmes en apposition signalés par des virgules, tirets d'incise et parenthèses à l'occasion desquels l'auteur ajoute des informations à propos des actants. Le lecteur est parfois obligé de revenir en arrière pour retrouver le fil directeur d'un passage. Et le traducteur? Il doit évidemment veiller non seulement à la compréhension exacte du texte mais à la bonne transposition des idées et du style vers la langue cible. Au regard des traductions analysées, une chose est claire: l'ordre des mots dans la traduction vers la langue cible doit être maintenu impérativement pour éviter la désagrégation de la cohérence syntaxique, ce qui a pour résultat une accumulation de subordonnées, surtout relatives en traduction slovène. Dans le groupe estudiantin qui a participé 
à ce projet, tous les traducteurs qui ont cherché à procéder d'une autre manière n'ont pas réussi à rendre les phrases intelligibles.

Le système de ponctuation slovène diffère du système français, le premier étant considéré comme un système syntaxique, le second comme un système déterminé par le sens ${ }^{19}$. La différence majeure porte sur l'emploi de la virgule: en slovène, la virgule est obligatoire dans la subordination ainsi que dans la coordination, tandis que ce n'est pas toujours le cas en français. Les autres signes de ponctuation, comme les points-virgules, les deux points et les parenthèses, peuvent être employés avec les mêmes valeurs en français et en slovène, ce qui permet leur simple transposition d'une langue à l'autre. Telle a été la stratégie retenue dans la plupart des traductions. Dans le texte du Tramway, le problème est compliqué puisque Simon déroge à la règle française et à ses normes. La discussion s'est donc engagée sur chaque exemple précis.

\section{Du nominal au verbal}

Une autre anomalie de traduction a reposé sur le glissement du verbal au nominal: Simon utilise volontiers des noms et des adjectifs signifiant l'apparence, souvent modalisée par les verbes être et sembler. Il a également recours à l'adjectif "semblable" (" un semblable et tragique visage", p. 63), mais aussi à des noms comme "la vue ", "l'aspect de quelque chose ». Dans la plupart des traductions vers le slovène, cette notion a été rendue par le verbe d'état, "voir " ou " paraître ": videti, zdeti se. Le caractère verbal du slovène s'oppose au français plus nominal. Mais ici encore les étudiants ont pris conscience que le mode de relation aux actants du récit est un peu transformé dans la traduction.

\section{BILAN DE L'EXPÉRIENCE}

Le bilan de l'analyse des traductions a montré que tout se joue autour de la phrase et des actants - si les étudiants ont pu identifier les bons actants, ils ont pu rétablir le texte de C. Simon dans leur propre langue. Faute de cette identification la traduction reste incompréhensible. Il faut mentionner que l'extrait traduit en anglais n'a pu être exploré à fond dans ce contexte et qu'il n'a servi qu'à titre de vérificateur pour une observation contrastive rapide. Sur les vingt-et-une traductions vers le slovène, et en fonction des critères mentionnés ci-dessus, trois

19. À comparer: J. Popin, La Ponctuation, Nathan Université, 1996; Slovenski pravopis, Ljubljana, SAZU, DZS, 2001. 
traductions ont été complètement inadéquates, quatre particulièrement réussies et les autres moyennes.

Globalement, le texte présenté comme exercice de traduction était d'une extrême difficulté. Les problèmes exposés au niveau stylistique par les traducteurs relèvent par excellence du style simonien privilégiant une phrase longue coupée de digressions qui exige une grande rigueur dans la traduction.

Parmi les autres difficultés rencontrées, nous pouvons citer l'absence de contexte général et la méconnaissance du contexte socioculturel de l'époque donnée. Le manque de connaissances plus précises sur la période du roman (la France provinciale des années 20, la Grande Guerre, la Seconde Guerre mondiale, etc.) empêchaient parfois les étudiants engagés dans la traduction de voir les liens et l'interaction globale entre les actants majeurs du texte. Le temps prévu pour l'exercice ne permettait qu'un apport limité d'informations.

La tendance des étudiants a été surtout de retenir le premier sens d'un terme complexe et polysémique, ainsi que de proposer, par le choix des mots en slovène, une interprétation, voire une explication psychologique des phénomènes. L'oeuvre de Simon en revanche ne la propose presque jamais. Si son approche est difficile, c'est peut-être avant tout parce que le récit est porté par un narrateur qui se garde de pénétrer dans le for intérieur des actants. La non-compréhension de cette démarche du romancier peut aboutir à une traduction mot-à-mot qui ne restitue pas le sens du texte.

Grosso modo, la complexité de cette écriture a outrepassé chez les participants toutes les attentes de lecture et de traduction. Et cette complexité a fait partie de l'expérience. L'exercice s'est certainement avéré utile et enrichissant, ne serait-ce que dans la mesure où il a permis à une nouvelle génération de futurs traducteurs un premier contact avec l'œuvre simonienne - à laquelle ils pourront revenir de manière plus avisée à l'avenir. 